\title{
Atrapamiento de la arteria poplítea: Comunicación de un caso
}

\author{
Juan Parra G, Felipe Corvalán Z, Fernando Ibáñez C, \\ Víctor Bianchi Sch, W aldo Bastías, Cristian Salas del C, \\ Alfredo Cam L, Andrés Lamarca T. \\ Popliteal artery entrampment. Report \\ of one case
}

Popliteal artery entrampment is a rare condition, occurring in young subjects, that causes ischemia of the extremity. The anatomical background is the compression or occlussion of the popliteal artery caused by forced plantar or dorsal flexion. We report a 32 year-old sportsman who presented with gangrene of the right first toe and a history of three months of progressive claudication. The Ankle-Brachial index and pulse volume curve registries showed a severe ischemia below the knee. Angiography showed a medial deviation of the popliteal artery associated to stenosis and aneurysm formation. A CT scan of the contralateral artery was normal. The patient was operated using a posterior approach, performing a reverse saphenous vein bypass graft and excising the popliteal artery. The postoperative period was uneventful (Rev Méd Chile 2006; 134: 207-10).

(Key w ords: Angiography; Popliteal artery; Vascular occlusion)

Recibido el 15 de julio, 2004. Aceptado el 13 de septiembre, 2005.

Servicio de Cirugía, Departamento de Cirugía Vascular, Hospital del Salvador, Santiago de Chile.

$\mathrm{E}^{1}$ atrapamiento de la arteria poplítea es una causa rara de claudicación o de isquemia aguda en personas jóvenes ${ }^{1-4}$. Esta entidad se origina en una alteración anatómica, que consiste en el paso de la arteria poplítea medial a la cabeza del gastrocnemio medial, produciendo así una compresión mecánica del vaso ${ }^{1,2,5}$. Dicha anormalidad anatómica fue descrita por primera vez en 1879 por un estudiante de medicina ${ }^{6}$; sin embar-

Correspondencia a: Dr. Juan Parra G. Departamento de Cirugía Vascular, Servicio de Cirugía, Hospital del Salvador. Av. Salvador 364, Providencia, Santiago de Chile. Tel: 3404392. Fax: 2040086. E mail: jparra@csm.cl go, sólo en 1959 fue reconocido como entidad clínica ${ }^{7}$.

La compresión crónica de la arteria poplítea daña sus paredes, pudiendo producir engrosamiento de la capa media, daño intimal, trombosis y formación de aneurismas. Si no es reconocido a tiempo, puede llevar a amputación de la extremidad por gangrena. Es por esto que su diagnóstico y tratamiento oportuno son de gran importancia para el paciente.

\section{CASO CLÍNICO}

Varón, 32 años de edad, deportista, con historia de 3 meses de evolución de claudicación intermiten- 
te. Consultó, al cabo de estos tres meses, por necrosis del primer dedo de su pie derecho.

En el examen físico destacó ausencia de pulsos poplíteo y distales en la extremidad comprometida y necrosis seca del tercio distal del $1^{\text {er }}$ dedo derecho. El examen de los pulsos en la extremidad contralateral fue normal.

Se realizó un registro de volumen de pulso en la extremidad derecha, evidenciándose alterado de rodilla a distal. El valor del índice tobillo-brazo fue 0,64 .

Se realizó angiografía por acceso femoral izquierdo, la cual mostró una medialización de la arteria poplítea derecha, asociada a estenosis irregular del lumen arterial y dilatación postestenótica de la arteria (Figura 1).

Se procedió a cirugía electiva, abordando el hueco poplíteo por vía posterior. Durante la disección de la estructuras vasculares se observó la arteria poplítea desplazada hacia medial por la cabeza medial del gastrocnemio (Figura 2). Se realizó resección del segmento comprometido (el cual se envió a estudio histológico) y reconstrucción mediante interposición de vena safena externa invertida. En el postoperatorio inmediato el paciente recuperó los pulsos poplíteo y tibial posterior.
El informe de anatomía patológica reveló ateroesclerosis hialina con formación de un aneurisma. El estudio con angiografía por tomografía axial computada (TAC) no reveló atrapamiento de la arteria poplítea contralateral.

El paciente evolucionó favorablemente sin progresión de la necrosis. Requirió una amputación del primer dedo, a nivel de la falange proximal, a los 3 meses del alta.

\section{DisCUSIÓN}

La claudicación de origen vascular en pacientes jóvenes es infrecuente y obliga a descartar etiologías tales como ateroesclerosis precoz, enfermedad quística adventicial, embolia no reconocida y atrapamiento de la arteria poplítea, entre las más frecuentes $^{2,5}$. El número de casos de pacientes con atrapamiento de la arteria poplítea ha aumentado en varios reportes extranjeros, probablemente por el mayor índice de sospecha que tienen los especialistas en medicina del deporte, quienes tienen la oportunidad de estudiar a los pacientes con dolor de pantorrilla ${ }^{2,5,8}$.

En el estudio no invasivo de estos pacientes están descritos la ecografía doppler o el doppler

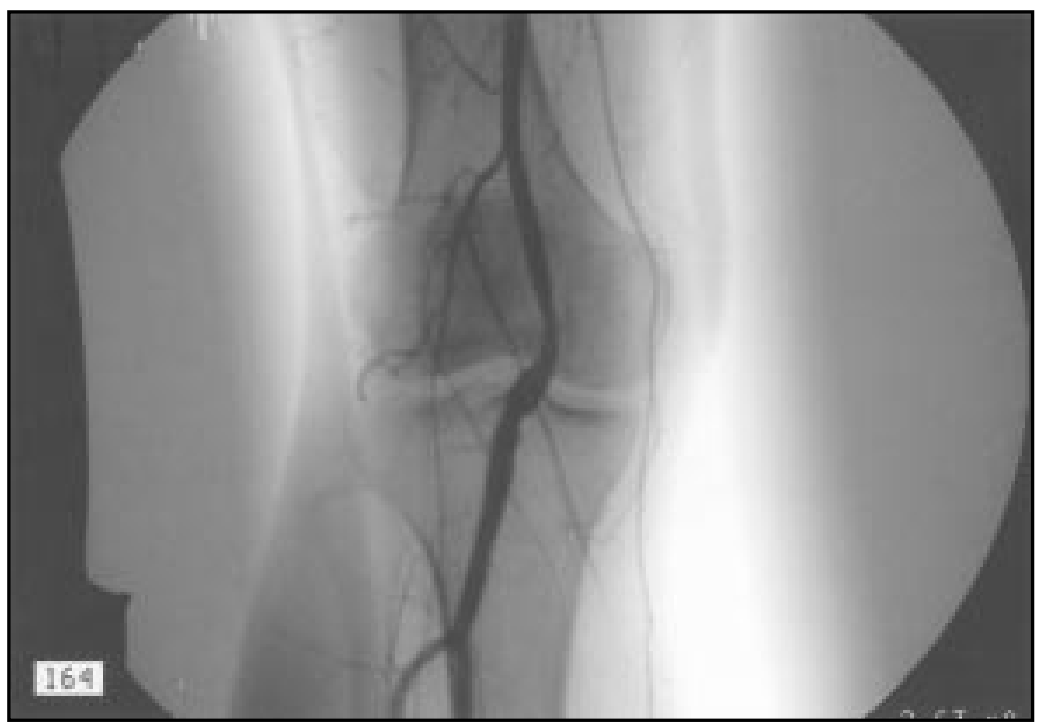

Figura 1. Arteriografía región poplítea. Nótese la desviación medial de la arteria, asociada a estenosis y formación de aneurisma postestenótico. 


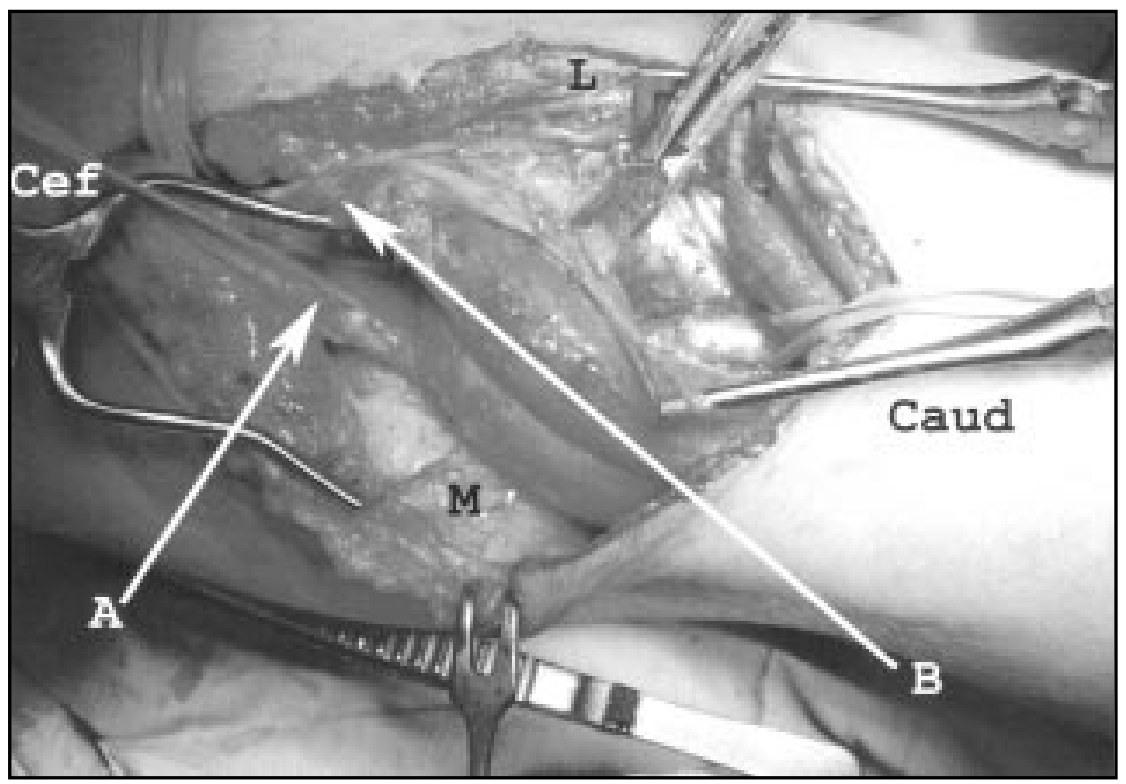

Figura 2. Fotografía intraoperatoria. A: Arteria poplítea. B: Cabeza del gastrocnemio medial. Cef: Cefálico. Cau: Caudal. M: Medial. L: Lateral. Nótese arteria poplítea pasando medial a la cabeza medial del gastrocnemio.

continuo con maniobras pasivas de dorsiflexión o flexión plantar activa, TAC de la fosa poplítea, resonancia magnética y test de esfuerzo $0^{1,9,10}$. La angiografía biplanar con maniobras de flexoextensión continúa siendo el estándar de oro para su diagnóstico, especialmente cuando se presentan signos de isquemia crítica o existe sospecha de degeneración de la pared de la arteria o formación de aneurisma ${ }^{1,11}$.

Una vez hecho el diagnóstico, el tratamiento es quirúrgico y su objetivo es disminuir el riesgo de isquemia de la extremidad, dada por trombosis de una arteria poplítea o por embolización distal de material trombótico proveniente de ésta. Ello depende principalmente de las alteraciones en la arteria poplítea o la presencia de obstrucción funcional. En este último caso, la literatura concuerda en realizar solamente la miotomía de la cabeza medial del gastrocnemio y otras bandas fibrosas que puedan existir. En el caso de trombosis, formación de aneurismas o alteración de la pared de la arteria poplítea, la interposición de safena es la alternativa más usada, dado que presenta resultados superiores cuando se compara con la miotomía sola. En la literatura se describen tanto el abordaje medial como el posterior, obteniendo este último una mejor visualización de las estructuras de la fosa poplítea ${ }^{1,2,11}$. El abordaje medial está asociado a una recuperación más rápida en pacientes jóvenes y es preferido por algunos autores ${ }^{2}$.

En nuestro paciente, quien se presentó como una isquemia crítica de la extremidad, la angiografía demostró la presencia de trombosis y formación de aneurisma de la arteria poplítea distal. El estudio contralateral se realizó con TAC de la fosa poplítea. Se realizó reemplazo del segmento arterial afectado por vena safena externa mediante abordaje posterior. Esta conducta diagnósticaterapéutica, vista a la luz de la literatura, representa un adecuado algoritmo de manejo para estos pacientes. 


\section{REFERENCIAS}

1. Levien LJ. Popliteal artery entrampment syndrome. Semin Vasc Surg 2003; 16: 223-31.

2. LeVIEN LJ, VeLLeR MG. Popliteal artery entrampment syndrome: More common than previously recognized. J Vasc Surg 1999; 30: 587-98.

3. ERdoes LS, Devine JJ, Bernhard VM, MD, BaKer MR, Berman SS, Hunter GC. Popliteal vascular compression in a normal population. J Vasc Surg 1994; 20: 978-86.

4. LAmberT AW, WiLKins DC. Popliteal artery entrampment syndrome. Br J Surg 1999; 86: 1365-70.

5. Arko FR, Harris EJ, Zarins CK, Olcott C. Vascular complications in high-performance athletes. J Vasc Surg 2001; 33: 935-42.
6. Stuart T. A note on a variation in the course of the popliteal artery. J Anat Physiol 1879; 13: 162.

7. HAMMING JJ. Intermittent claudication at an early age, due to an anomalous course of the popliteal artery. Angiology 1959; 10: 369-71.

8. Stager A, Clement D. Popliteal artery entrampment syndrome. Sports Med 1999; 2: 61-70.

9. Wimams LR, Funn WR, McCarthy WJ, Yao JST, Bergan JJ. Popliteal artery entrampment: Diagnosis by computed tomography. J Vasc Surg 1986; 3: 360-3.

10. Di Marzo L, Cavalaro A, Sciacca V, Lepidi S, Marmorale A, Tamburew A et al. Diagnosis of popliteal artery entrampment syndrome: The role of duplex scanning. J Vasc Surg 1991; 13: 434-8.

11. Cowns PS, McDonald PT, Lim RC. Popliteal artery entrampment: An evolving syndrome. J Vasc Surg 1989; 10: 484-90. 